\title{
Linearly chirped fiber Bragg grating response to thermal gradient: from bench tests to the real-time assessment during in vivo laser ablations of biological tissue
}

Paola Saccomandi

Ambra Varalda

Riccardo Gassino

Daniele Tosi

Carlo Massaroni

Michele A. Caponero

Raoul Pop

Sanzhar Korganbayev

Guido Perrone

Michele Diana

Alberto Vallan

Guido Costamagna

Jacques Marescaux

Emiliano Schena 


\title{
Linearly chirped fiber Bragg grating response to thermal gradient: from bench tests to the real-time assessment during in vivo laser ablations of biological tissue
}

\author{
Paola Saccomandi, ${ }^{\mathrm{a}, \mathrm{b}, *}$ Ambra Varalda, ${ }^{\mathrm{b}}$ Riccardo Gassino, ${ }^{\mathrm{c}}$ Daniele Tosi, ${ }^{\mathrm{d}, \mathrm{e}}$ Carlo Massaroni ${ }^{\mathrm{b}}{ }^{\mathrm{b}}$ \\ Michele A. Caponero, ${ }^{f}$ Raoul Pop, ${ }^{g}$ Sanzhar Korganbayev, ${ }^{\mathrm{d}}$ Guido Perrone,${ }^{\mathrm{c}}$ Michele Diana, ${ }^{\mathrm{a}, \mathrm{h}}$ \\ Alberto Vallan, ${ }^{\mathrm{c}}$ Guido Costamagna, ${ }^{\mathrm{a}, \mathrm{i}}$ Jacques Marescaux, ${ }^{\mathrm{a}, \mathrm{h}}$ and Emiliano Schena ${ }^{\mathrm{b}}$ \\ a IHU-Strasbourg - Institute of Image-Guided Surgery, Strasbourg, France \\ bUniversità Campus Bio-Medico di Roma, Unit of Measurements and Biomedical Instrumentation, Rome, Italy \\ 'Politecnico di Torino, Department of Electronics and Telecommunications, Torino, Italy \\ ${ }^{\mathrm{d} N a z a r b a y e v}$ University, School of Engineering, Astana, Kazakhstan \\ eNazarbayev University, National Laboratory Astana, Biosensors and Bioinstrumentation Laboratory, Kazakhstan \\ ${ }^{\mathrm{f}}$ Research Centre of Frascati, Photonics Micro-and Nano-Structures Laboratory, Agenzia nazionale per le nuove tecnologie, L'energia e lo sviluppo \\ economico sostenibile, Frascati, Italy \\ ${ }^{9} \mathrm{CHRU}$-Centre Hospitalier Régional et Universitaire, Strasbourg, France \\ hIRCAD-Research Institute against Cancer of Digestive System, Strasbourg, France \\ 'Università Cattolica del Sacro Cuore, Digestive Endoscopy, Policlinico Gemelli, Rome, Italy
}

\begin{abstract}
The response of a fiber optic sensor [linearly chirped fiber Bragg grating (LCFBG)] to a linear thermal gradient applied on its sensing length (i.e., $1.5 \mathrm{~cm}$ ) has been investigated. After these bench tests, we assessed their feasibility for temperature monitoring during thermal tumor treatment. In particular, we performed experiments during ex vivo laser ablation (LA) in pig liver and in vivo thermal ablation in animal models (pigs). We investigated the following: (i) the relationship between the full width at half maximum of the LCFBG spectrum and the temperature difference among the extremities of the LCFBG and (ii) the relationship between the mean spectrum wavelength and the mean temperature acting on the LCFBG sensing area. These relationships showed a linear trend during both bench tests and LA in animal models. Thermal sensitivity was significant although different values were found with regards to bench tests and animal experiments. The linear trend and significant sensitivity allow hypothesizing a future use of this kind of sensor to monitor both temperature gradient and mean temperature within a tissue undergoing thermal treatment. (๑) 2017 Society of Photo-Optical Instrumentation Engineers (SPIE) [DOI: 10.1117/1.JBO.22.9.097002]
\end{abstract}

Keywords: chirped fiber Bragg grating; laser ablation; in vivo study; thermal measurement.

Paper 170321R received May 18, 2017; accepted for publication Aug. 17, 2017; published online Sep. 14, 2017.

\section{Introduction}

Over the last decade, fiber Bragg grating (FBG) sensor technology has gained popularity in several fields, such as health monitoring, impact detection, automotive, medical applications, and physiological monitoring. ${ }^{1,2}$ The reason behind this is that FBG sensors offer major advantages over other sensors developed with a different technology, including immunity from electromagnetic fields, a rapid response, high sensitivity, and multiplexing capabilities. ${ }^{1,3}$

The interest in FBG sensors is strictly related to their sensitivity to strain and temperature, as well as the possibility to provide distributed measurement. ${ }^{2,4}$ A number of applications, especially in the medical field, can take advantage of this feature. For instance, hyperthermal procedures used for the treatment of tumors are a case in point of a specific field where temperature monitoring may be particularly beneficial to improve clinical outcomes. ${ }^{5,6}$ A number of studies have been conducted to investigate the possibility of using FBGs in this

*Address all correspondence to: Paola Saccomandi, E-mail: paola saccomandi@ihu-strasbourg.eu context. In the late 1990s, Rao et al. developed a FBG-based system for temperature monitoring during hyperthermia procedures. The system was tested inside a 4.7-T MR scanner: the probe revealed a temperature resolution of $0.2^{\circ} \mathrm{C}$ and an accuracy of $0.8^{\circ} \mathrm{C}$, in a range of $25^{\circ} \mathrm{C}$ to $60^{\circ} \mathrm{C}$, and the system was also tested in vivo. ${ }^{2}$ Over the last few years, the use of FBG in temperature monitoring during hyperthermal treatment has gained large interest as testified by several articles published in this topic. ${ }^{2,7,8}$ During hyperthermia procedures, the temperature of the tumor is strongly increased, to achieve protein denaturation and to lead to the controlled necrosis of the malignant mass. In this application, the temperature must be raised over $100^{\circ} \mathrm{C}$ in the region of the tissue close to the energy delivery system [e.g., fiber optic applicator in case of laser ablation (LA)]. ${ }^{9}$ The phenomena of heat conduction and blood perfusion affect the treatment, because tissue temperature decreases at a certain distance from the applicator. The thermal gradient, which in some procedures can be extremely high (up to $50^{\circ} \mathrm{C} / \mathrm{mm}^{9}$ ), has a strong effect on outcome. Subsequently, it requires accurate monitoring. Uniform FBGs provide one

$1083-3668 / 2017 / \$ 25.00$ @ 2017 SPIE 
temperature value, which is mostly related to the temperature averaged on the sensitive length of the grating. As a result, when there is a high thermal gradient, the length of the FBGs can influence the accuracy of the measurement, ${ }^{10,11}$ and ideally punctual gratings can provide the real value of the tissue temperature in a specific place. To have the tissue temperature distributed around the applicator may be particularly beneficial in providing direct insights into the thermal gradient. Although linearly chirped fiber Bragg gratings (LCFBGs) can provide such information, only a few studies involving their use may be found in the literature ${ }^{5,12-14}$ Different from uniform FBGs, the characterization of LCFBGs for thermal gradient measurement is not straightforward, and a preliminary analysis is mandatory to find a correlation between the spectrum features and the thermal gradient. ${ }^{12,15}$ The aim of this article is to fill the gap in the literature by performing a careful assessment of LCFBG response to thermal gradients, under various conditions. First, a constant temperature was applied along the active length, to perform the static calibration of the sensor. Second, the sensor was subjected to linear thermal gradients during bench tests. Finally, the feasibility assessment of LCFBG for temperature monitoring during LA of ex vivo organs was performed. In these experiments, the temperature was simultaneously monitored by means of thermocouples and array of uniform FBGs. Finally, LCFBG was used in a preclinical scenario, i.e., to monitor tissue temperature during LA of liver in in vivo animal models (pigs).

\section{Principle of Work of Fiber Bragg Grating Sensors}

The working principle of FBGs is related to their specific characteristics and to their fabrication process. ${ }^{3,4,6,16}$ Specifically, an FBG is written into a single-mode fiber, which presents a periodic variation of the refraction index, ${ }^{17}$ obtained by exposing the core of the fiber to an intense optical interference pattern. The possibility to reproduce permanent gratings in an optical fiber was demonstrated in 1978 by Hill and Meltz. ${ }^{18}$ Nowadays, the holographic technique and the phase mask technique are the most effective methods used to inscribe Bragg gratings in photosensitive fibers. ${ }^{19,20}$ Specifically, the core of the fiber is exposed to an ultraviolet beam that can locally change the refractive index proportionally to the incident energy. The resulting grating reflects light of a specific wavelength, called Bragg wavelength $\left(\lambda_{\mathrm{B}}\right)$, depending on the spacing of the periodic variation and on the modulation of the refractive index. Consequently, an FBG acts as a filter that conveys all the wavelengths that are not in resonance with it and reflects the ones that follow the Bragg condition, given as

$\lambda_{B}=2 \cdot \eta_{\mathrm{eff}} \cdot \Lambda$,

where $\eta_{\mathrm{eff}}$ is the effective refractive index of the fiber core and $\Lambda$ is the period of index modulation. ${ }^{21}$ When a broadband light source propagates within the fiber, a narrow spectral component centered in $\lambda_{\mathrm{B}}$ is reflected by the grating.

One of the most interesting configurations is the LCFBG. These sensors show a monotonically increasing grating period along the sensing element ( $z$-axis of the fiber). The consequence is that $\lambda_{\mathrm{B}}$ changes along the $z$-axis

$\lambda_{\mathrm{B}}(z)=2 \cdot \eta_{\mathrm{eff}} \cdot \Lambda(z)$.

Regarding LCFBGs, the relationship between $\Lambda$ and $z$ is linear and can be expressed as

$\Lambda(z)=\Lambda_{0}+k \cdot z$

with $0<z<L$, where $L$ is the length of the grating and $k$ is the chirp rate coefficient, which defines the increase in the refractive index period along the optical fiber, ${ }^{5}$ and $\Lambda_{0}$ is the period at one of the two extremities of the grating (in $z=0$ ).

As a result, $\lambda_{\mathrm{B}}$ linearly changes along the $z$-axis

$\lambda_{\mathrm{B}}(z)=2 \cdot \eta_{\mathrm{eff}} \cdot\left(\Lambda_{0}+k \cdot z\right)$.

The spectrum of the reflected light has a width that is larger than uniform FBG. The typical commercial length of LCFBGs ranges from 1.5 to $5 \mathrm{~cm}$ and the spectrum bandwidth from 5 to $50 \mathrm{~nm}^{5}$

A typical resulting spectrum is shown in Fig. 1(a).

An external stimulus (temperature or strain) applied to the active part of the sensor causes a change in the reflected
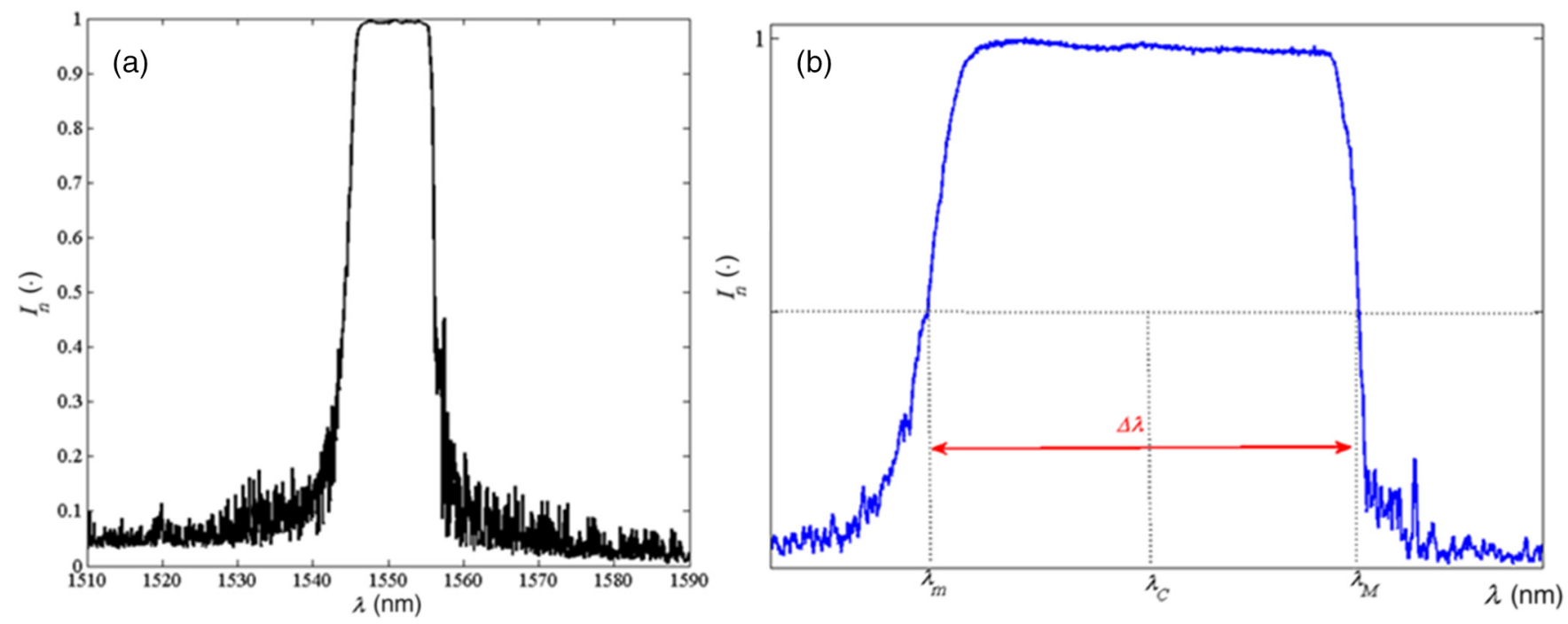

Fig. 1 (a) Typical normalized spectrum obtained by interrogating an LCFBG and (b) example of a normalized spectrum acquired during the test, in which $\Delta \lambda, \lambda_{C}, \lambda_{m}$, and $\lambda_{M}$ parameters are shown. 
spectrum. In particular, the application of a temperature variation $(\Delta T)$ at each point of the active region of the sensor entails a shift of the Bragg wavelength, $\Delta \lambda_{\mathrm{B}}$, which can be expressed as

$\Delta \lambda_{\mathrm{B}}(z)=S_{T} \cdot \Delta T(z)$,

where $S_{T}$ is the thermo-optic coefficient.

Equation 5 shows a linear relationship between each portion of the grating and the variation of the corresponding local temperature. $^{11,22}$

\section{Static Calibration of the Linearly Chirped Fiber Bragg Grating Sensor: Experimental Setup and Results}

Section 3.1 is focused on the description of the experimental setup used for the static calibration of the LCFBG sensor under test. In Sec. 3.2, the results are shown.

\subsection{Static Calibration: Experimental Setup}

The LCFBG sensor under test (model FBG A141111-017, Technica SA, $1.5 \mathrm{~cm}$ of active length, $1550 \pm 0.5 \mathrm{~nm}$ of central wavelength of, $10 \mathrm{~nm}$ of $3-\mathrm{dB}$ bandwidth, reflectivity $>90 \%$, which is embedded into a single-mode SMF-28e fiber with a $250-\mu$ m-diameter acrylate coating, was calibrated. During the experiments, the LCFBG was interrogated by a Bragg meter interrogator (FiberSensing, BraggMETER, FS2200) based on a scanning laser source. The reference temperature was measured with a thermocouple (type T, RS Pro, accuracy $= \pm 0.5^{\circ} \mathrm{C}$ ), placed close to the active length of the LCFBG. The calibration was performed on a thermostatic chamber (PN120, Carbolite). The chamber temperature was set at $100^{\circ} \mathrm{C}$. The chamber was then turned off when the temperature target was reached. During the slow cooling phase, which lasted about $6 \mathrm{~h}$, the spectrum of the LCFBG was collected with a sample frequency of $1 \mathrm{~min}$. During this phase, the temperature shifted from $100^{\circ} \mathrm{C}$ to about $25^{\circ} \mathrm{C}$.

\subsection{Static Calibration: Data Analysis and Results}

For each acquired spectrum, we followed the two following steps: (i) each spectrum was normalized $\left(I_{n}\right)$, (ii) from each normalized spectrum, the $\lambda_{C}$ average wavelength was estimated by calculating the mean value between the value of $\lambda_{m}$ and of $\lambda_{M}$, which represent the minimum and the maximum wavelength at 0.5 amplitude of the spectrum, as shown in Fig. 1(b), and (iii) each spectrum was acquired at a specific temperature, $T$, which was measured with the thermocouple. Consequently, the values of $\lambda_{C}$ calculated for each spectrum was related to the temperature value provided by the thermocouple at the same instant of analyzed spectrum acquisition. The relationship between $\lambda_{C}$ and $T$ is shown in Fig. 2 .

As shown in Fig. 2, the relationship between $\lambda_{C}$ and $T$ is well represented with a linear model. Consequently, we calculated the calibration curve of the LCFBG as the best-fitting line between $\lambda_{C}$ and $T$. The appropriate agreement between the linear model and the experimental data is confirmed by the high value of the correlation coefficient $\left(R^{2}=0.99\right)$ and by the low value of the root-mean-square error $(\mathrm{RMSE}=8.2 \mathrm{pm})$. The sensitivity of the LCFBG was calculated as the slope of the best-fitting line $\left(10.4 \mathrm{pm} /{ }^{\circ} \mathrm{C}\right)$.

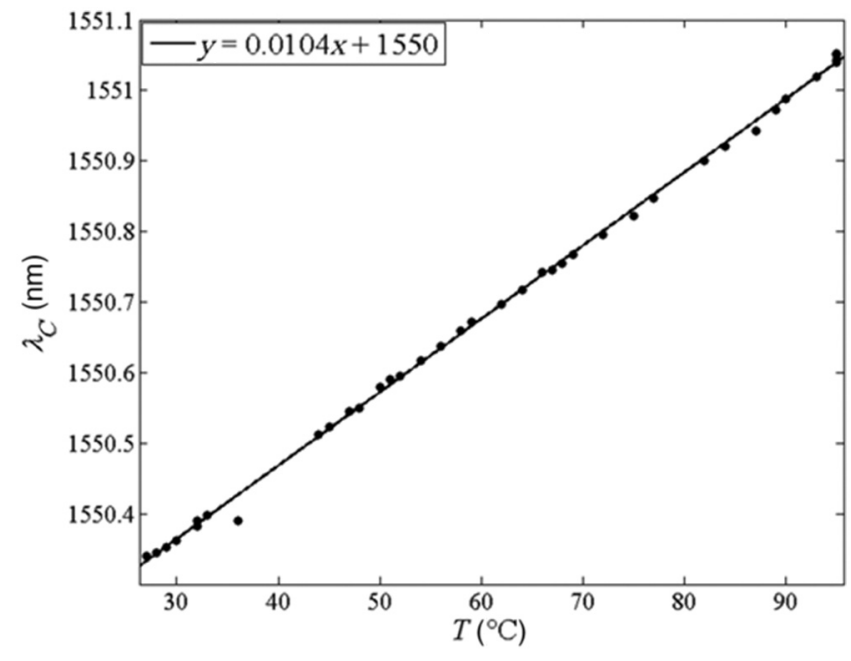

Fig. 2 Correlation between the average wavelength of the LCFBG and the temperature. Experimental data (black dots) and the best-fitting line (continuous black line) are shown.

\section{Response of Linearly Chirped Fiber Bragg Grating to Linear Thermal Gradient: Bench Tests}

In this section, the experimental setup was prepared to apply a linear thermal gradient on the active area of the LCFBG, and the results obtained during the experiments are shown.

\subsection{Linearly Chirped Fiber Bragg Grating Response to Linear Gradient: Experimental Setup}

The experimental setup shown in Fig. 3 allowed us to apply a linear thermal gradient on the sensor under test. A brass plate

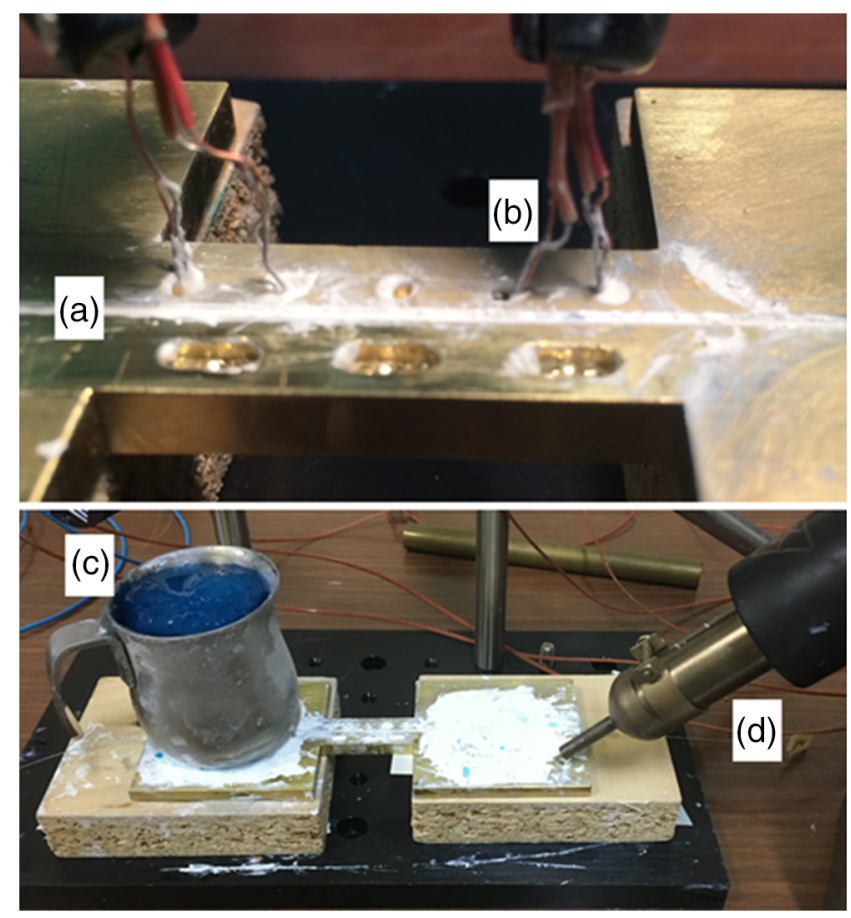

Fig. 3 Experimental setup prepared to apply a linear thermal gradient on the LCFBG sensor: (a) depth groove along the length of the plate where the sensor is placed in contact with the brass, (b) thermocouples used to measure the brass temperature along the sensor active area, (c) box containing ice, and (d) hot air welder. 
$(15 \mathrm{~cm} \times 6 \mathrm{~cm} \times 0.5 \mathrm{~cm})$ was used for this scope. A $1-\mathrm{mm}$ depth groove along the length of the plate was made to position the sensor in contact with the brass [Fig. 3(a)]. The LCFBG under test was placed along the groove with its active length positioned in correspondence to the thinning of the brass plate, and one extremity of the sensor was connected to a Bragg meter interrogator (FiberSensing, BraggMETER, FS2200). Four circular holes were carved on the plate for the placement of four thermocouples [type T, RS Pro, accuracy $= \pm 0.5^{\circ} \mathrm{C}$, Fig. 3(b)] used to monitor the temperature along the sensor during the test. The holes are $0.5-\mathrm{cm}$ distant from each other and very close to the central groove (i.e., $1 \mathrm{~cm}$ ) to minimize the temperature difference between the one measured with the thermocouples and the temperature acting on the sensing area of the LCFBG. The thermocouple outputs were recorded with the NI 9211 module. To reproduce a linear thermal gradient along the active area of the sensor, a box containing ice kept at $-70^{\circ} \mathrm{C}$ [Fig. 3(c)] and a hot air welder [HTC 900, Fig. 3(d)] were placed over the brass plate, as shown in Fig. 3.

With this configuration, one extremity of the sensor is subjected to a low temperature given by the ice action, while the other extremity is under a higher temperature caused by the welder activity. A linear thermal gradient was obtained with a maximum difference of approximately $30^{\circ} \mathrm{C}$ between the two extremities.

\subsection{Linearly Chirped Fiber Bragg Grating Response to Linear Gradient: Results}

The experimental setup described in Sec. 4.1 allowed us to apply a linear thermal gradient along the active area of the sensor. Figure 4(a) shows the temperature trend along the $z$-axis, which was recorded with the four thermocouples during four instants of the experiments. Of note, the extremity of the LCFBG closer to the box containing ice $(z=0 \mathrm{~cm})$ is always subjected to a lower temperature as compared to the other extremity of the sensor, which is closer to the welder $(z=1.5 \mathrm{~cm})$.

To analyze the response of the LCFBG to a linear thermal gradient, both the average wavelength $\left(\lambda_{C}\right)$ and the full width at half maximum (FWHM, $\Delta \lambda$ ) were calculated. The first parameter was obtained as outlined in Sec. 3.2 and in Fig. 1(b), while $\Delta \lambda$ was calculated as the difference between $\lambda_{M}$ and $\lambda_{m}$ [see Fig. 1(b)]. First, $\lambda_{C}$ was related to the average temperature $\left(T_{\text {mean }}\right)$ applied along the active area of the sensor $\left(T_{\text {mean }}\right.$ is calculated as the average of the temperatures recorded with the four thermocouples placed along the active area of the sensor); $\Delta \lambda$ was then related to the thermal gradient $\Delta T$ (estimated by the difference between the two thermocouples placed at the two extremities of the active area). Figures 4(b) and 4(c) show these trends.

Both the relationships shown in Figs. 4(b) and 4(c) are well represented with a linear model $\left(R^{2}=0.988\right.$ and 0.993 , respectively, and RMSE $=21$ and $7.0 \mathrm{pm}$, respectively). Sensitivity values, estimated as the slope of the best-fitting line, were as follows: 9.5 and $4.5 \mathrm{pm} /{ }^{\circ} \mathrm{C}$, respectively.

(a)

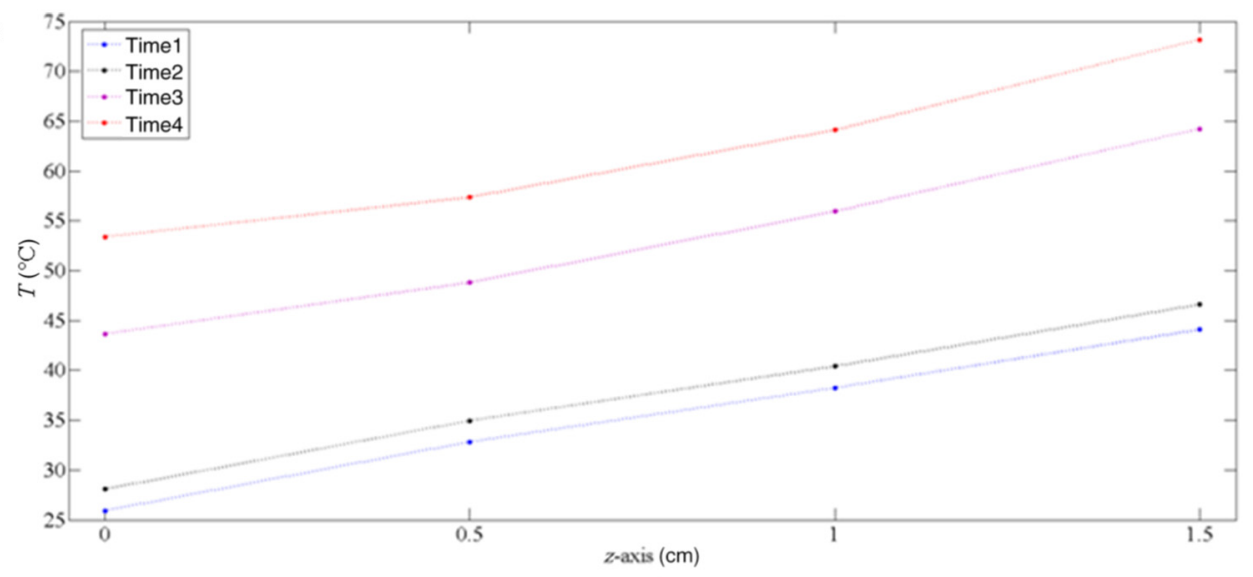

(b)
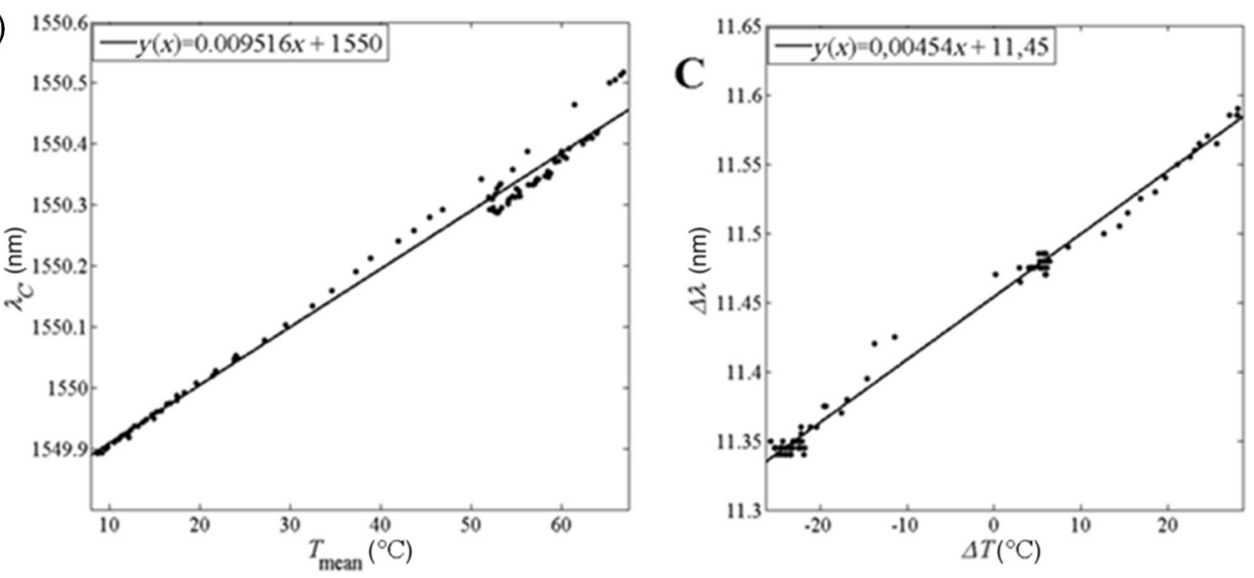

Fig. 4 (a) Temperature gradient measured with the four thermocouples on the active area of the sensor, (b) average wavelength versus mean temperature acting on the LCFBG, and (c) FWHM versus temperature difference between the extremities of the LCFBG. 


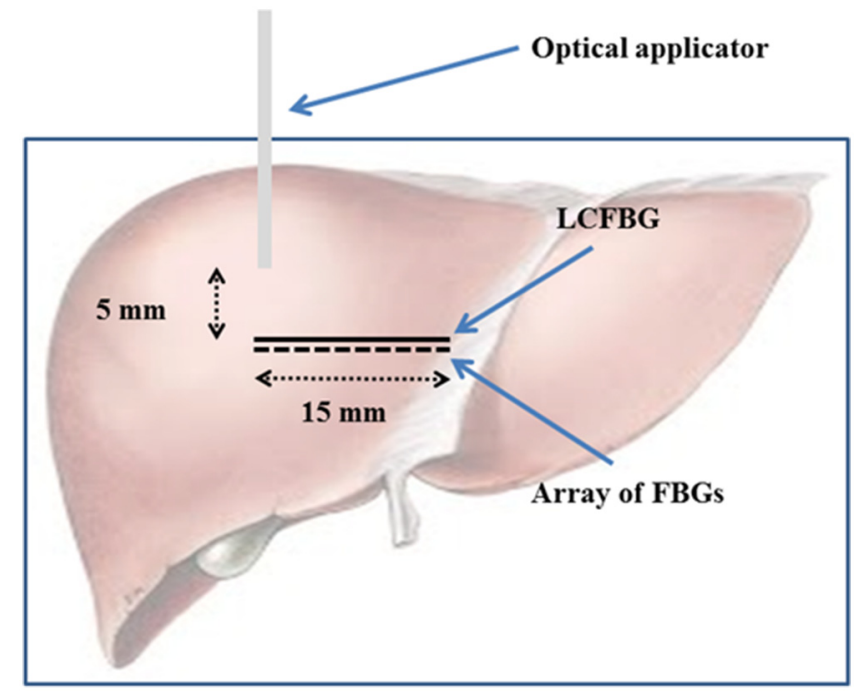

Fig. 5 Schematic representation of the configuration used during ex vivo LA.

\section{Experiments in Ex Vivo Animal Models Undergoing Laser Ablation}

In this section, the feasibility assessment of LCFBG for temperature monitoring during LA was investigated in ex vivo animal models (pig livers). The experimental setup and the results are presented.

\subsection{Linearly Chirped Fiber Bragg Grating Response During Laser Ablation in Ex Vivo Animal Models: Experimental Setup}

To assess the feasibility of LCFBG for temperature monitoring during LA, we performed experiments in ex vivo animal models. The laser light (Nd:YAG, wavelength of $1064 \mathrm{~nm}$ ) was set at a power of $3 \mathrm{~W}$, and treatment time was $3 \mathrm{~min}$. The laser applicator was inserted within the animal organ, perpendicular to the LCFBG sensor, at a distance of $\sim 5 \mathrm{~mm}$ and centered to one of the two extremities of the LCFBG. This configuration allows to have a temperature gradient along the active area of the LCFBG: the extremity close to the applicator will reach a higher temperature as compared to the other extremity. To have a reference measurement of the temperature on the active area of the LCFBG, nine uniform FBG sensors were positioned on a fiber placed in contact to the LCFBG, to cover the entire LCFBG length, as schematically shown in Fig. 5. Two arrays for a total of nine uniform FBGs are housed inside two fibers with one lying over the other one, and the arrays are inscribed inside an acrylate SMF-28e fiber, with external diameter of $0.25 \mathrm{~mm}$. The first fiber embeds an array of two gratings, with sensitive length of $1 \mathrm{~mm}$, at a relative distance of $15 \mathrm{~mm}$. The Bragg wavelengths of the gratings at room temperature are 1540
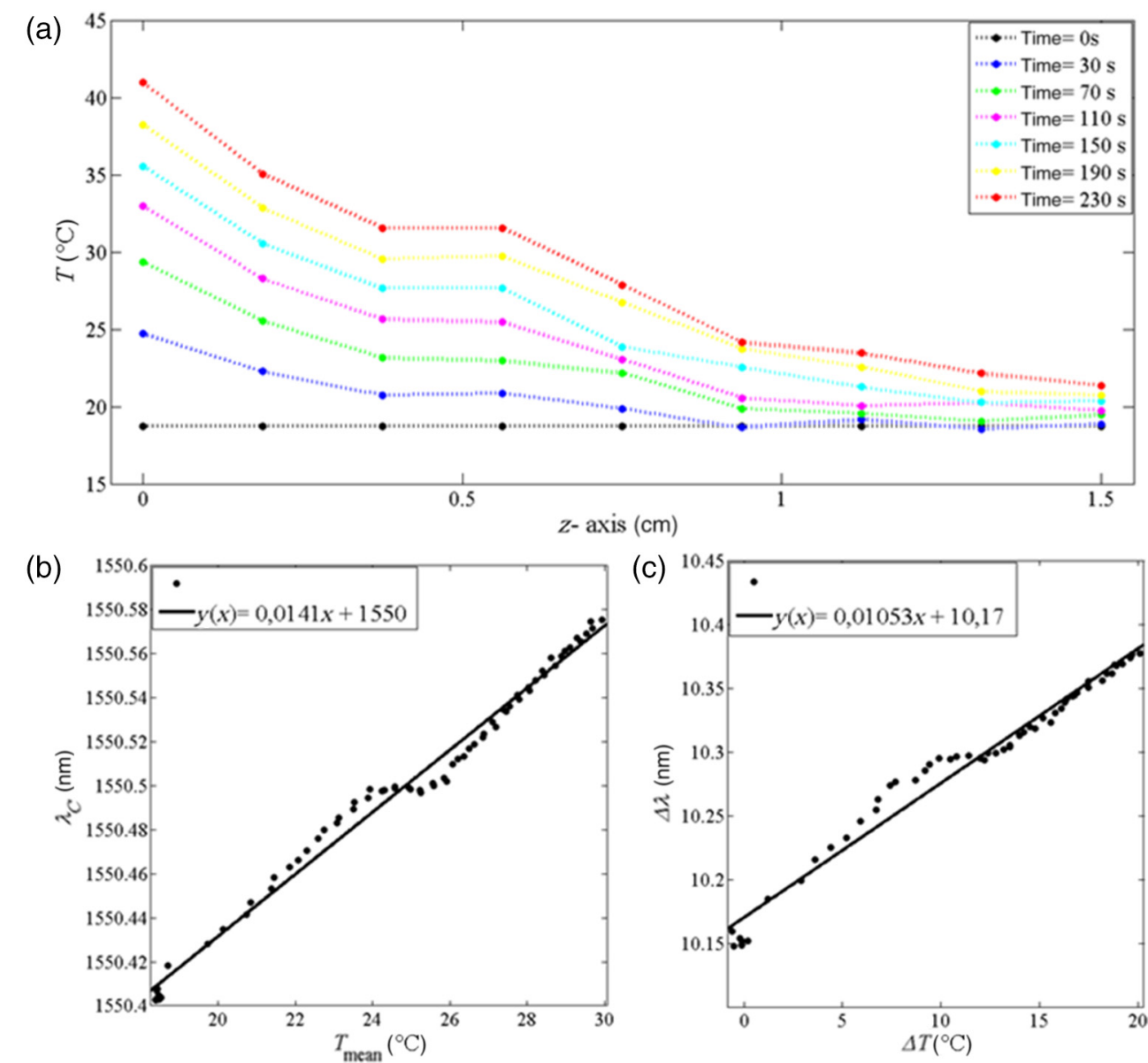

Fig. 6 (a) Temperature trend along the $z$-axis of the sensor and (b) average wavelength versus average temperature. Data and the best-fitting line are shown. (c) FWHM versus temperature difference between the extremities of the LCFBG active area. Data and the best-fitting line are shown. 
and $1550 \mathrm{~nm}$. The second FBG array is constituted by seven gratings, each with a sensitive length of $1 \mathrm{~mm}$, at a relative distance of $1 \mathrm{~mm}$. The Bragg wavelengths of the seven gratings at room temperature ranged from 1530 to $1560 \mathrm{~nm}$, with a step of about $5 \mathrm{~nm}$ between each peak. For these small sized gratings, the reflectivity ranges between $35 \%$ and $44 \%$.

\subsection{Linearly Chirped Fiber Bragg Grating Response During Laser Ablation in Ex Vivo Animal Models: Results}

To estimate the temperature trend along the $z$-axis of the sensor, the initial temperature of the organ $\left(\sim 18.7^{\circ} \mathrm{C}\right)$ was measured with a thermocouple before starting the LA. Figure 6(a) shows the temperature on the active area of the LCFBG at different instants of time, which was estimated by the nine FBGs. As could be observed in Fig. 6(a), the temperature gradient in a tissue undergoing LA is not linear, because of the mechanisms of laser light absorption and scattering in the region of the tissue proximal to the applicator. ${ }^{23}$

The $\lambda_{C}$ and $\Delta \lambda$ parameters were calculated as described in Fig. 1. Afterward, $\lambda_{C}$ was related to $T_{\text {mean }}$ (calculated as the mean temperature of the nine FBGs used as a reference), while $\Delta \lambda$ was related to $\Delta T$ (which calculated the difference between the temperature measured with the two FBGs placed at the extremities of the LCFBG). Figures 6(b) and 6(c) show these trends $\left(\lambda_{C}\right.$ versus $T_{\text {mean }}$ and $\Delta \lambda$ versus $\Delta T$, respectively). They are well represented via a linear model $\left(R^{2}=0.986\right.$ and 0.973 , respectively, and RMSE $=6.0$ and $11 \mathrm{pm}$, respectively). Sensitivity values, estimated as the slope of the best-fitting lines, are about 14.1 and $10.5 \mathrm{pm} /{ }^{\circ} \mathrm{C}$ respectively.

Experiments in in vivo animal models undergoing LA

The last test was carried out to assess the feasibility of LCFBG for temperature monitoring in in vivo animal models. In particular, the LCFBG response was acquired during LA on the liver of a living pig. The experimental setup and the results are presented.

\subsection{Linearly Chirped Fiber Bragg Grating Response During Laser Ablation in an In Vivo Animal Model: Procedure}

Thermal treatment was performed in a pig liver with a laser diode (power set at $5 \mathrm{~W}$ and treatment time: $10 \mathrm{~min}$ ), according to an experimental protocol that received the full approval from the Institutional Ethical Committee (Protocol No. $38.2015 .01 .069)$. The pig (55 kg, male) was anesthetized using $10 \mathrm{cc}$ of Propofol and $5 \mathrm{cc}$ of Esmeron, and 2\% isoflurane was injected during the ongoing procedure. The basal temperature of the animal $\left(38^{\circ} \mathrm{C}\right)$ was measured with a thermometer for clinical use. The procedure and the placement of the laser applicator and fiber optic sensors were performed percutaneously by an expert radiologist and guided through computed tomography (CT) imaging. Two conventional surgical needles were used to insert both the laser applicator and the gratings inside the in vivo liver. The procedure was the following: (i) the CT exam was performed in order to choose the suitable parenchymal region of the liver (absence of big blood vessels, inside on the lobe) in which to perform LA and (ii) the surgical needle guiding the laser applicator was inserted inside the chosen region. First, the radiologist inserted the needle in the animal liver; second, he introduced the laser fiber inside the needle, and finally he retracted the needle, to leave inside the organ only the fiber optic applicator with the emitting surface in direct contact with
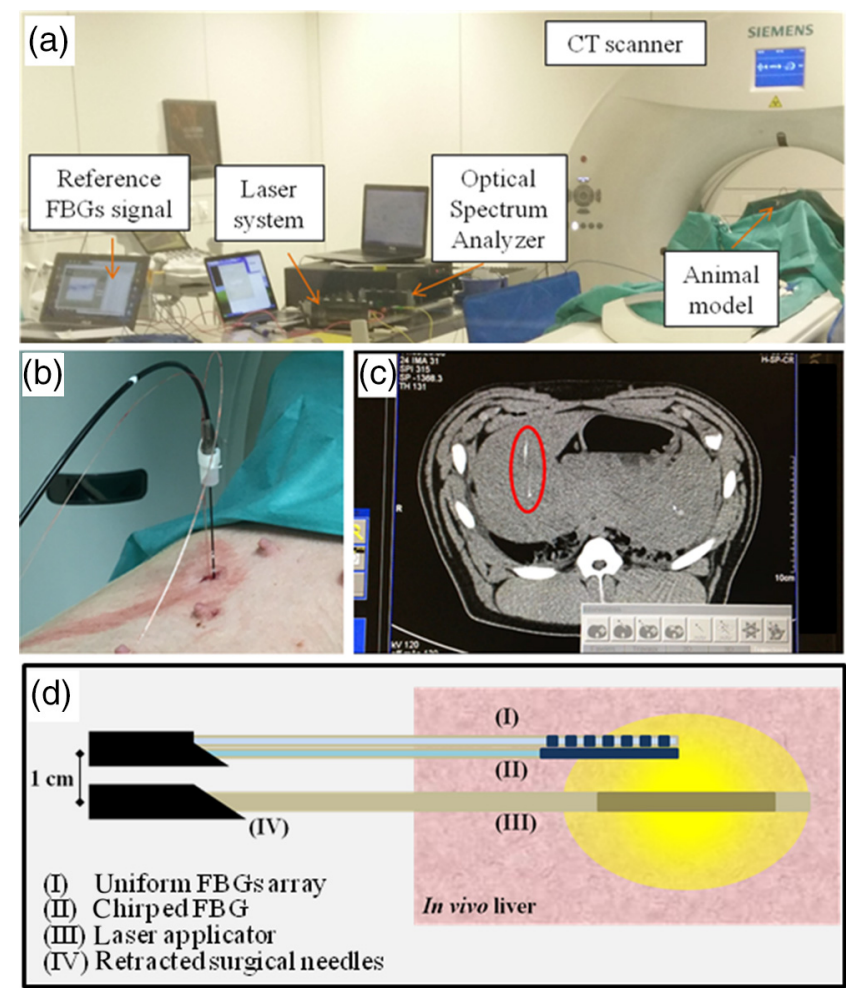

Fig. 7 (a) Scenario of the experimental trials, (b) percutaneous insertion of the optical applicator and of sensors within the surgical needles, (c) CT image used to guide the needle (highlighted with a red ellipse) within the liver, and (d) schematic of the relative positions between the gratings (I and II) and the laser applicator (III), and a detail of the surgical needles that are retracted (IV) to leave the gratings in contact with the ablated tissue region (distances and lengths are not in scale).

the parenchyma; (iii) a further CT scan was performed to check the actual position of the applicator and to define the trajectory and the distance of the second needle for the insertion of the gratings in the organ. The desired position is the grating needle parallel to the fiber applicator, with an axial distance of $1 \mathrm{~cm}$, and the tip of the FBGs placed at the same height of the emission center of the fiber applicator; (iv) the above-mentioned needle is placed inside the animal to target the organ, and two optical fibers (one housing the uniform seven FBGs array, and the other housing the chirped grating) are inserted inside this needle. The two FBGs fibers are in-built; (v) one last CT scan is acquired to verify that the desired relative positioning between the sensors and the applicator has been accomplished and (vi) the needle used to guide the gratings inside the organ is then retracted, aiming to leave the FBGs in direct contact with the tissue undergoing the laser treatment.

Figure 7 shows the in vivo experimental setup, with details of the CT operation room [Fig. 7(a)], the percutaneous insertion of the needles [Fig. 7(b)], the CT image scanned after the placement of the fibers within the liver [Fig. 7(c)], and the schematic of the relative distance among the gratings and the laser applicator [Fig. 7(d)].

\subsection{Linearly Chirped Fiber Bragg Grating Response During Laser Ablation in an In Vivo Animal Model: Results}

Figure 8 shows the trends of the temperature gradient acting on the chirped and measured in four different times during the LA 

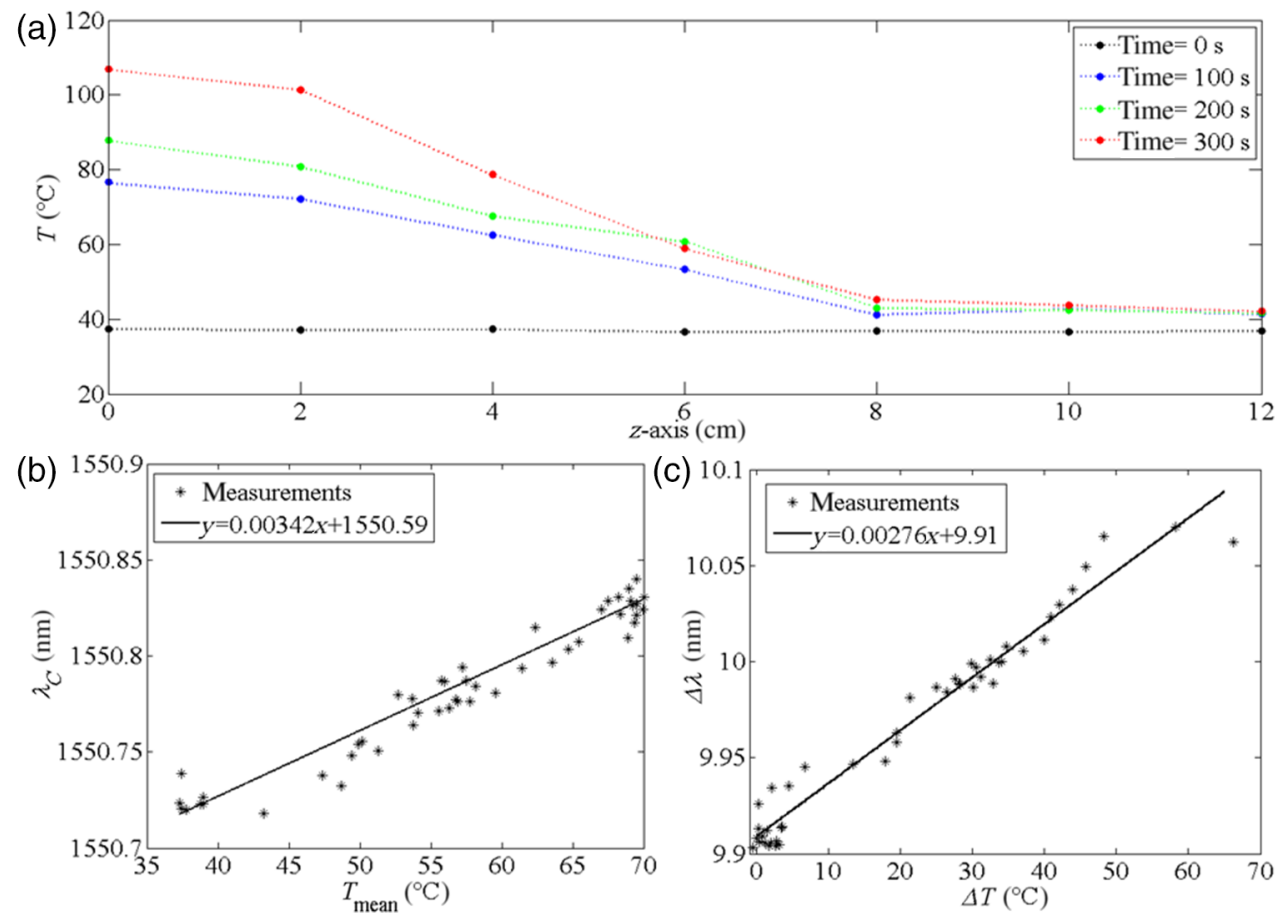

Fig. 8 (a) Temperature gradient measured on the chirped FBG, at different times during the ablation procedure, (b) average wavelength versus average temperature, and (c) FWHM versus temperature difference between the extremities of the LCFBG active area. In both graphs, data and the best-fitting line are shown.

procedure, $\lambda_{C}$ and $\Delta \lambda . \lambda_{C}$ and $\Delta \lambda$ constitute the functions of $T_{\text {mean }}$ and $\Delta T$, respectively. The reference temperatures have been measured with an array of FBGs, placed adjacent to the LCFBG, as described in Sec. 5.2.

The relationships shown in Figs. 8(a) and 8(b) $\left(\lambda_{C}\right.$ versus $T_{\text {mean }}$ and $\Delta \lambda$ versus $\Delta T$, respectively) are well represented with a linear model $\left(R^{2}=0.944\right.$ and 0.957 , respectively, and RMSE $=8.9$ and $9.0 \mathrm{pm}$, respectively). Sensitivity values, estimated as the slope of the best-fitting lines, are $\sim 3.4$ and $2.8 \mathrm{pm} /{ }^{\circ} \mathrm{C}$, respectively.

\section{Discussion and Conclusions}

The aim of this study was twofold: to investigate the response of an LCFBG to a linear thermal gradient and to perform a feasibility assessment of this type of sensor for temperature monitoring in biological tissues undergoing LA.

The measurement of tissue temperature increase during thermal treatment is pivotal to guarantee the adequate and desired outcome of the therapy, i.e., to thermally damage the malignant tissue and to spare the surrounding healthy anatomical structures from risky temperature increase. Several studies demonstrated that fiber optic sensors, and FBG in particular, can appropriately accomplish this task; being small and flexible, these sensors are easy to insert inside organs. ${ }^{24}$ Additionally, FBGs allow for the simultaneous measurement of temperature in different positions along only one fiber. ${ }^{6,11}$ LCFBGs provide an essential additional feature for this specific application, namely, the possibility to measure thermal gradients in real time. The correlation between the experienced thermal gradient and spectrum change is a demanding task, which requires a preliminary analysis. ${ }^{5}$

In our work, we moved from the bench trial to the direct application of the measuring system in a test performed in an in vivo animal model. Our results demonstrated the feasibility of LCFBGs for the monitoring of the thermal gradient and mean temperature along its active area. We performed this assessment in considering both linear gradient (during bench test) and nonlinear, yet monotonically increasing thermal gradients in tissue undergoing LA in both ex vivo and in vivo animal models. The parameters used to estimate the temperature difference between the two extremities of the LCFBG $(\Delta T)$ and the mean temperature $\left(T_{\text {mean }}\right)$ acting on the sensor were the FWHM $(\Delta \lambda)$ and the mean spectrum wavelength $\left(\lambda_{C}\right)$, respectively.

Results showed that both the $\Delta \lambda$ versus $\Delta T$ relationship and $\lambda_{C}$ versus $T_{\text {mean }}$ are well represented with a linear model as testified by the high value of $R^{2}$ and the low values of RMSE.

If considering the experimental results obtained through the bench test analysis, when the LCFBG experienced a linear thermal gradient, of the relationship $\lambda c$ versus $T_{\text {mean }}$ is linear, and the sensitivity (i.e., $0.00951 \mathrm{~nm} /{ }^{\circ} \mathrm{C}$ ) is close to the expected numerical sensitivity (i.e., $0.0102 \mathrm{~nm} /{ }^{\circ} \mathrm{C}$ ), calculated by the model developed in Refs. 15 and 25.

On the other hand, the $\Delta \lambda$ versus $\Delta T$ trend provides a slight disagreement between simulations and experimental data. Indeed, while the $\Delta \lambda(\Delta T)$ trend is linear, for linear gradients, the sensitivity exhibits a difference: it is equal to $0.0081 \mathrm{~nm} /{ }^{\circ} \mathrm{C}$ in the simulations, while this is equal to $0.0045 \mathrm{~nm} /{ }^{\circ} \mathrm{C}$ in experiments. This can be explained in part with the nonideality of the LCFBG spectrum used in measurements compared with the simulated CFBG spectrum.

The bigger dispersion of the data obtained during in vivo experiments is demonstrated by the lower values of $R^{2}$ and the bigger RMSE values with respect to the ones obtained during bench trials and ex vivo experiments. This result can be related to the breathing movements of the animal, which can cause small but not negligible strains to the LCFBG. We also want to point out the different values of thermal sensitivity 
estimated as the slope of the best-fitting lines during the bench tests, the ex vivo experiments, and the in vivo trials (see Figs. 4, 6 , and 8 ). These distinct values may be generated by the different boundary conditions of all the tests, by the different shapes of the gradient, but especially by a potential misalignment between the LCFBG and the temperature sensors used as a reference. Indeed, during bench tests, although the experimental conditions were highly controlled, it is worth saying that the LCFBG was in contact with the brass plate only in his lower surface, while the upper side was in contact with the thermal paste and the room temperature. Conversely, in the ex vivo and in vivo tests, the sensor is completely surrounded by tissue, and this condition may slightly influence the response of the grating.

Moreover, during bench tests, the relative position among the reference sensors and the LCFBG can be adjusted accurately. On the other hand, principally in in vivo experiments, the positioning is not straightforward, because both the LCFBG and the reference sensors (array of FBGs) are manually inserted within the organ. This maneuver did not allow the operator to perform an accurate adjustment of the relative positioning among the sensors.

Another significant cause of this misalignment may be the displacement of the gratings due to the breathing movements of the animal. We have previously proved that the maximum displacement of the liver during the respiration can bring a measurement error of a about $2^{\circ} \mathrm{C}$ on uniform $\mathrm{FBG},{ }^{26}$ but no data are available regarding the entity of this strain error on the LCFBG. In addition, we should include the hypothesis that the sensors slightly change their positions during the test.

Since the thermal gradient is significant, the inaccurate positioning can lead to a high difference between the temperature used as a reference (estimated by the array of FBGs) and the temperature acting on the LCFBG. For instance, the temperature gradient measured in vivo through the uniform FBG array has a maximum value of $70^{\circ} \mathrm{C}$ on $13-\mathrm{mm}$ length; hence, being the thermal gradient equal to $5.4^{\circ} \mathrm{C} / \mathrm{mm}$, a potential misalignment of $4.6 \mathrm{~mm}$ can entail a temperature difference up to $25^{\circ} \mathrm{C}$.

In light of these considerations, further analyses should be anticipated to disseminate the approach, to assess the potential measurement error due to breathing movement on the LCFBG, as well as to evaluate the sensitivity of the LCFBGs to a nonlinear thermal gradient.

\section{Disclosures}

The authors have no relevant financial interests in this article and no potential conflicts of interest to disclose.

\section{Acknowledgments}

The authors would like to thank Mr. Mourad Bouhadjar and the technicians' team for the use of CT-scanner at IHU Strasbourg. The authors also thank Mr. Guy Temporal and Mr. Christopher Burel for their assistance in proofreading the article. This research was partially supported by IHU, Institute of ImageGuided Surgery of Strasbourg, under grant I-Thermo LAP.

\section{References}

1. H. Ling et al., "Viability of using an embedded FBG sensor in a composite structure for dynamic strain measurement," Measurement 39(4), 328-334 (2006).

2. Y. J. Rao, "Recent progress in applications of in-fiber Bragg grating sensors," Opt. Laser Eng. 31(4), 297-324 (1999).
3. A. D. Kersey et al., "Fiber grating sensors," J. Lightwave Technol. 15(8), 1442-1463 (1997).

4. X. Bao and L. Chen, "Recent progress in distributed fiber optic sensors," Sensors 12(7), 8601-8639 (2012).

5. D. Tosi et al., "Fiber-optic chirped FBG for distributed thermal monitoring of ex-vivo radiofrequency ablation of liver," Biomed. Opt. Express 5(6), 1799 (2014).

6. E. Schena et al., "Fiber optic sensors for temperature monitoring during thermal treatments: an overview," Sensors 16(7), 1144 (2016).

7. W. W. Morey, G. Meltz, and W. H. Glenn, "Fiber optic Bragg grating sensors," Proc. SPIE 1169, 98-107 (1989).

8. E. Schena et al., "Experimental assessment of CT-based thermometry during laser ablation of porcine pancreas," Phys. Med. Biol. 58(16), 5705-5716 (2013).

9. J. A. Pearce, J. W. Valvano, and S. Emelianov, "Temperature measurements," in Optical-Thermal Response of Laser-Irradiated Tissue, pp. 399-453, Springer, The Netherlands (2011).

10. P. Saccomandi et al., "Influence of FBG sensors length on temperature measures in laser-irradiated pancreas: theoretical and experimental evaluation," in 35th Annual Int. Conf. of the IEEE Engineering in Medicine and Biology Society (EMBC '13), pp. 3737-3740 (2013).

11. R. Gassino et al., "Characterization of fiber optic distributed temperature sensors for tissue laser ablation," in IEEE Int. Instrumentation and Measurement Technology Conf. (I2MTC '17), Torino (2017).

12. F. Napoleoni et al., "Monitoring of thermal treatment by linearly chirped fiber Bragg grating sensors: feasibility assessment during laser ablation on ex vivo liver," in 38th Annual Int. Conf. of the IEEE Engineering in Medicine and Biology Society (EMBC'16), pp. 6493-6496 (2016).

13. D. Tosi, E. G. Macchi, and A. Cigada, "Fiber-optic temperature and pressure sensors applied to radiofrequency thermal ablation in liver phantom: methodology and experimental measurements," J. Sens. 2015, 909012 (2015).

14. K. Fröjdh, G. Hedin, and S. Helmfrid, "Strain and temperature measurement using a 9.5-m continuous chirped fiber Bragg grating with millimeter resolution," in 25th IEEE Optical Fiber Sensors Conf. (OFS'17) (2017).

15. S. Korganbayev et al. "Linearly chirped fiber-optic Bragg grating as distributed temperature sensor for laser ablation," in IEEE SENSORS, pp. 1-3 (2016).

16. F. Taffoni et al., "Optical fiber-based MR-compatible sensors for medical applications: an overview," Sensors 13(10), 14105-14120 (2013).

17. Y.J. Rao et al., "Optical in-fiber Bragg grating sensor system for medical applications,” J. Biomed. Opt. 3(1), 38-44 (1998).

18. K. O. Hill and G. Meltz, "Fiber Bragg grating technology fundamentals and overview," J. Lightwave Technol. 15(8), 1263-1276 (1997).

19. B. A. Tahir, J. Ali, and R. A. Rahman, "Fabrication of fiber grating by phase mask and its sensing applications," J. Optoelectron. Adv. Mater. 8(4), 1604-1609 (2006).

20. Z. Zhou and J. Ou, "Development of FBG sensors for structural health monitoring in civil infrastructures," in Sensing Issues in Civil Structural Health Monitoring, pp. 197-207, Springer, The Netherlands (2005).

21. S. J. Mihailov, "Fiber Bragg grating sensors for harsh environments," Sensors 12(2), 1898-1918 (2012).

22. E. G. Macchi et al., "Optical fiber sensors-based temperature distribution measurement in ex vivo radiofrequency ablation with sub millimeter resolution," Biomed. Opt. Express 19(11), 117004 (2014).

23. P. Saccomandi et al., "Theoretical analysis and experimental evaluation of laser-induced interstitial thermotherapy in ex vivo porcine pancreas," IEEE Trans. Biomed. Eng. 59(10), 2958-2964 (2012).

24. N. T. Pham et al., "Real-time temperature monitoring with fiber Bragg grating sensor during diffuser-assisted laser-induced interstitial thermotherapy," J. Biomed. Opt. 22(4), 045008 (2017).

25. D. Tosi et al., "Towards inline spatially resolved temperature sensing in thermal ablation with chirped fiber Bragg grating," in IEEE Int. Symp. on Medical Measurements and Applications (MeMeA), Benevento, Italy (2016).

26. C. Cavaiola et al., "Error of a temperature probe for cancer ablation monitoring caused by respiratory movements: ex vivo and in vivo analysis," IEEE Sens. J. 16(15), 5934-5941 (2016).

Paola Saccomandi, $\mathrm{PhD}$, is a postdoctoral research fellow at IHU-Strasbourg, and an assistant professor at Università Campus 
Bio-Medico di Rome, where she received her PhD in biomedical engineering in 2014. Her main research interests include the study and development of sensors and image-based tools for the monitoring physiological parameters, and temperature change during hyperthermia procedures for cancer treatment.

Ambra Varalda received her MS degree in biomedical engineering in 2015 from Università Campus Bio-Medico di Roma, Italy. Her master thesis was focused on the monitoring temperature effect during hyperthermia procedures by means of linear chirped fiber Bragg grating sensors.

Riccardo Gassino received his MS degree in biomedical engineering in 2015 from Politecnico di Torino, Italy, where he is currently pursuing his $\mathrm{PhD}$ in metrology, working on the development of innovative sensing systems for biomedical applications.

Daniele Tosi, PhD, is an assistant professor in electrical and electronic engineering at Nazarbayev University, and head of Biosensors and Bioinstruments Laboratory at National Laboratory Astana. Previously, he was Marie Curie fellow at University of Limerick. His research interest include fiber optic sensors and biosensors, biomedical sensors, and sensing networks. He is an associate editor of IEEE Sensors Journal.

Carlo Massaroni, $\mathrm{PhD}$, is postdoctoral research fellow at Università Campus Bio-Medico di Roma. He received his BEng and MEng degrees and his $\mathrm{PhD}$ in biomedical engineering from Università Campus Bio-Medico di Roma, in 2010, 2012 and 2016, respectively. His main research interests include the development of systems for monitoring parameters of physiological interest and the design, development, and test of optic-based sensors for mechanical and thermal measurements.

Michele A. Caponero is a senior researcher at ENEA, the Italian National Agency for New Technologies, Energy and Economic Sustainable Development. $\mathrm{He}$ is member of the working group TC86C "Fibre optic systems and active devices-Fibre optic sensors" of IEC, the International Electrotechnical Commission. His research activity is mainly focused on the development of sensing systems based of fibre optic technology for applications in civil and geotechnical engineering, nuclear physics, cultural heritage conservation, and biomedical applications.

Raoul Pop, MD, is an interventional radiologist at the Centre Hospitalier Régional et Universitaire of Strasbourg, and is specialized in neurointerventional therapies. His research domains also include visceral vascular and percutaneous interventions and in particular bariatric embolisation.

Sanzhar Korganbayev received his BS degree in electrical and electronic engineering from Nazarbayev University, Astana, Kazakhstan, in 2016. He is currently working toward MS degree at Nazarbayev University and working as a research assistant in Biosensors and Bioinstruments Laboratory at National Laboratory Astana. His research interests include fiber optic sensors and their applications for thermal and mechanical measurements.

Guido Perrone received his $\mathrm{PhD}$ in electromagnetics/photonics from the Politecnico di Torino, Torino, Italy, where he is a professor of microwave systems and of optical devices. He is the author of more than 200 publications in international journals and conferences. His current research interests include fiber optic sensors and highpower lasers for industrial and biomedical applications.

Michele Diana, MD, PhD, is a director of the Research Unit on EndoLaparoscopic Procedures at the IHU-Strasbourg Institute of ImageGuided Surgery. His main research interest is related to image-guided therapies by various imaging modalities including fluorescence, confocal, hyperspectral, virtual and augmented reality. He is author of more than 100 publications.

Alberto Vallan is an associate professor in the Department of Electronics and Telecommunications of the Politecnico di Torino, Italy. From 2000 to present, he has been a lecturer at the Politecnico di Torino in courses concerning electronic measurements and sensors. His research interests are focused on the development and characterization of fiber sensors and measuring instruments for biomedical and industrial applications.

Guido Costamagna, MD, is a full professor of surgery and director in the Institute of Clinical Surgery at the Università Cattolica del Sacro Cuore, Rome. He is a head of the Department of Digestive and Endocrine-Metabolic Diseases and a director of Digestive Endoscopy Unit at Fondazione Policlinico Agostino Gemelli, Rome, as well as chair of excellence in Digestive Endoscopy, USIAS, University of Strasbourg, France. His main research interest are in the fields of digestive and therapeutic endoscopy.

Jacques Marescaux, MD, is a professor of surgery, chairman in the Institute of Image-Guided Surgery in Strasbourg. He is a president and founder of IRCAD France, Taiwan and América Latina. Those Institutes ensure the training of 8000 surgeons each year. He and his team published more than 4000 national and international articles and communications. Besides, he is a fellow of the American College of Surgeons (FACS) and the Royal College of Surgeons in London.

Emiliano Schena, PhD, is an associate professor in mechanical and thermal measurement at Università Campus Bio-Medico di Roma. His current research interests include sensors and transducers for mechanical and thermal measurements in the biomedical and clinical field, respiratory rehabilitation, as well as the design, development, and test of optic-based sensors for mechanical and thermal measurements. He is author of more than 150 publications. 